\title{
The Absolute Summability of Fourier Series.
}

\author{
By J. M. WhitTaker. \\ (Received 30th May 1929. Read 8th June 1929)
}

$\S 1 . \quad$ Absolute summability $(A)$.

An elementary proposition states that an absolutely convergent series is convergent, i.e. that if

$$
\left|s_{1}-s_{0}\right|+\left|s_{2}-s_{1}\right|+\ldots+\left|s_{n}-s_{n-1}\right|<K,(\text { all } n)
$$

then

$$
s_{n} \rightarrow \text { a limit, as } n \rightarrow \infty .
$$

This is the analogue for series of the theorem on functions that if a function $f(x)$ is of bounded variation in an interval, the limits $f(x \pm 0)$ exist at every point. Consider, in particular, the function

$$
f(x)=\sum_{0}^{\infty} a_{n} x^{n}
$$

the series being supposed convergent in $(0 \leqslant x<1)$. Then the theorem states that the Abel limit

$$
\lim _{x \rightarrow 1-0} f(x)
$$

exists provided that $f(x)$ is of bounded variation in $(0,1)$ so that

$$
\sum_{r=1}^{m}\left|f\left(x_{r}\right)-f\left(x_{r-1}\right)\right|<K
$$

for all subdivisions $0=x_{0}<x_{1}<x_{2}<\ldots<x_{m}<1$.

If the Abel limit exists $\Sigma a_{n}$ is said to be summable $(A)$. It is therefore natural to say that $\Sigma a_{n}$ is absolutely summable $(A)$ if $f(x)$ is of bounded variation in $(0,1)$. It will then be true that a series which is absolutely summable $(A)$ is also summable $(A)$.

By Abel's classical theorem, every convergent series is summable (A). It is easy to prove that every absolutely convergent series is absolutely summable (A). For the condition (2) is equivalent to

$$
\int_{0}^{1}\left|f^{\prime}(x)\right| d x \quad \text { exists }
$$


and if $\Sigma a_{n}$ is absolutely convergent

$$
\begin{aligned}
\int_{0}^{x_{1}}\left|f^{\prime}(x)\right| d x & \leqslant \int_{0}^{x_{1}}\left\{\sum_{1}^{\infty} n\left|a_{n}\right| x^{n-1}\right\} d x, \quad\left(0 \leqslant x_{1}<1\right) \\
& =\sum_{1}^{\infty}\left|a_{n}\right| x_{1}{ }^{n} \leqslant \sum_{1}^{\infty} \mid a_{n}
\end{aligned}
$$

and (3) is satisfied.

§2. Absolute summability $(A)$ of Fourier series.

The convergence of a Fourier series at a point depends only on the values of the function in the immediate neighbourhood of the point, but this is not true of absolute convergence. Thus it is known ${ }^{1}$ that unless a function is continuous in the whole interval $(-\pi, \pi)$, its Fourier series can only converge absolutely at the points of a set of measure zero. It is however true of absolute summability (A), and it will be shown that the Fourier series of an integrable function $f(\theta)$ is absolutely summable (A) at every point at which Dini's condition is satisfied, in particular at every point at which $f^{\prime}(\theta)$ exists. Thus, let

$$
\frac{1}{2} a_{0}+\sum_{n=1}^{\infty}\left(a_{n} \cos n \theta+b_{n} \sin n \theta\right)
$$

be the Fourier series of a function $f(\theta)$ which has a Lebesgue integral in $(-\pi, \pi)$ and let

$$
\phi(t)=\frac{f(\theta+2 t)+f(\theta-2 t)-2 l}{2}
$$

Then, (4) is absolutely summable $(A)$ to sum $l$ if

exists.

$$
\int_{0}^{\delta}\left|\frac{\phi(t)}{t}\right| d t
$$

In other words, every Fourier series which converges in virtue of Dini's condition is absolutely summable $(A)$.

The Poisson series ${ }^{2}$ (convergent for $0 \leqslant x<1$, since $a_{n}, b_{n} \rightarrow 0$ ) is

$$
\begin{aligned}
P(x) & =\frac{1}{2} a_{0}+\sum_{n=1}^{\infty} x^{n}\left(a_{1} \cos n \theta+b_{n} \sin n \theta\right) \\
& =\frac{1}{2 \pi} \int_{-\pi}^{\pi} f(\alpha)-\frac{1-x^{2}}{1-2 x \cos (\theta-\alpha)+x^{2}} d \alpha
\end{aligned}
$$

so that, writing $\alpha=\theta+2 t$,

${ }^{1}$ Hobson. Functions of a Real Variable. 2 (1926), 550.

2 ibid., 629. 


$$
Q(x)=P(x)-l=\frac{2}{\pi} \int_{0}^{\frac{\pi}{2}} \phi(t) \frac{1-x^{2}}{1-2 x \cos 2 t+x^{2}} d t .
$$

Then the total variation of $Q(x)$ in $\left(0, x_{1}\right)$ is

$$
\begin{aligned}
\int_{0}^{x_{1}}\left|Q^{\prime}(x)\right| d x & =\frac{2}{\pi} \int_{0}^{x_{1}}\left|\int_{0}^{\frac{\pi}{2}} \phi(t) \frac{d}{d x}\left\{\frac{1-x^{2}}{1-2 x \cos 2 t+x^{2}}\right\} d t\right| d x \\
& \leqslant-\frac{4}{\pi} \int_{0}^{x_{1}} d x \int_{0}^{\frac{\pi}{2}}|\phi(t)|\left|\frac{\left(1+x^{2}\right) \cos 2 t-2 x}{\left(1-2 x \cos 2 t+x^{2}\right)^{2}}\right| d t \\
& \leqslant \frac{2}{\pi} \int_{0}^{\frac{\pi}{2}}|\phi(t)| V\left(x_{1}, t\right) d t
\end{aligned}
$$

inverting the order of integration ${ }^{1}$, where

$$
V\left(x_{1}, t\right)=2 \int_{0}^{x_{1}}\left|\frac{\left(1+x^{2}\right) \cos 2 t-2 x}{\left(1-2 x \cos 2 t+x^{2}\right)^{2}}\right| d x .
$$

Let $t_{1}=\frac{1}{2} \arccos \frac{2 x_{1}}{1+x_{1}^{2}}=\operatorname{arccot} x_{1}-\frac{\pi}{4}, \quad\left(0<t_{1}<\frac{\pi}{4}\right)$.

Then if $0 \leqslant t \leqslant t_{1}$, and $p(x)=\frac{1-x^{2}}{1-2 x \cos 2 t+x^{2}}$,

$$
\begin{aligned}
V\left(x_{1}, t\right) & =2 \int_{0}^{x_{1}} \frac{\left(1+x^{2}\right) \cos 2 t-2 x}{\left(1-2 x \cos 2 t+x^{2}\right)^{2}} d x \\
& =\int_{0}^{x_{1}} p^{\prime}(x) d x \\
& =p\left(x_{1}\right)-1<p\left(x_{1}\right) \\
& \leqslant \frac{\sin 2 t_{1}}{1-\cos 2 t_{1}}=\cot t_{1}<\frac{\pi}{2 t_{1}} \leqslant \frac{\pi}{2 t}
\end{aligned}
$$

while if $\quad t_{1} \leqslant t \leqslant \frac{\pi}{4}$,

$$
\begin{aligned}
V\left(x_{1}, t\right) & =\int_{0}^{\cot \left(t+\frac{\pi}{4}\right)} p^{\prime}(x) d x-\int_{\cot \left(t+\frac{\pi}{4}\right)}^{x_{1}} p^{\prime}(x) d x \\
& =\frac{2}{\sin 2 t}-1-p\left(x_{1}\right)<\frac{2}{\sin 2 t} \\
& \leqslant \frac{\pi}{2 t} .
\end{aligned}
$$

1 Op. cit., 1 (1921), 577. 
Finally, if $\frac{\pi}{4} \leqslant t \leqslant \frac{\pi}{2}$

$$
V\left(x_{1}, t\right)=-\int_{0}^{x_{1}} p^{\prime}(x) d x<1 .
$$

Thus

$$
\begin{aligned}
\int_{0}^{x_{1}}\left|Q^{\prime}(x)\right| d x & \leqslant \frac{2}{\pi}\left(\int_{0}^{t_{1}}+\int_{t_{1}}^{\pi}+\int_{\frac{\pi}{4}}^{\frac{\pi}{2}}\right)|\phi(t)| V\left(x_{1}, t\right) d t \\
& <\int_{0}^{\pi} \frac{\mid \phi(t)}{t} d t+\frac{2}{\pi} \int_{\frac{\pi}{4}}^{\frac{\pi}{2}}|\phi(t)| d t
\end{aligned}
$$

so that $Q(x)$ is of bounded variation in $(0,1)$.

Dini's condition ${ }^{1}$ is not necessary for absolute summability (A), nor even for absolute convergence. For the series

$$
f(x)=\sum_{n=1}^{\infty} \frac{\cos 2^{n} x}{n^{*}}
$$

converges absolutely for all real values of $x$, but does not satisfy Dini's condition at $x=0$. To prove this, take $\delta=\pi 2^{-m}$, where $m$ is an integer. Then

$$
\begin{aligned}
\int_{\delta}^{\pi}\left|\frac{\phi(t)}{t}\right| d t & \left.=\int_{\delta}^{\pi} \sum_{n=1}^{\infty} \frac{1-\cos 2^{n+1} t}{n^{2}}\right) \frac{d t}{t} \\
& =2 \sum_{n=1}^{\infty} \frac{1}{n^{3}} \int_{\delta}^{\pi} \frac{\sin ^{2} 2^{n} t}{t} d t \\
& >2 \sum_{n=m+1}^{\infty} \frac{1}{n !} \int_{\pi^{2} 2^{n-m}}^{\pi \cdot 2^{n}} \frac{\sin ^{2} u}{u} d u \\
& >K \sum_{n=m+1}^{\infty} \frac{1}{n} \log 2^{m}>K \sqrt{ } m
\end{aligned}
$$

where $K$ is an absolute constant.

Again, a Fourier series may converge at a point without being absolutely summable (A). This is shown by the following example, suggested to me by Professor Littlewood. Let

$$
\frac{z(1-z)^{i}}{\log \frac{1}{1-z}}=\sum_{n=1}^{\infty} c_{n} z^{\prime \prime}
$$

I The remainder of the paper was added, 14th August 1929, to answer two questions raised by Dr Copson. 
It is clear that the series converges for $|z|<1$. Now let $\Gamma_{n}$ denote the contour in the $z$-plane consisting of the straight lines joining successive points $1+n^{-1}, n, n+n i,-n+n i,-n-n i$, $n-n i, n, 1+n^{-1}$, together with a circle of radius $n^{-1}$ round $z=1$. Then

$$
c_{n}=\frac{1}{2 \pi i} \int_{l^{\prime a}} \frac{(1-z)^{i}}{z^{n} \log \frac{1}{1-z}} d z
$$

and a straightforward calculation shows that

$$
n c_{n} \rightarrow 0 \text {. }
$$

Moreover

$$
\lim _{r \rightarrow 1-0} \frac{r e^{i \theta}\left(1-r e^{i \theta}\right)^{i}}{\log \frac{1}{1-r e^{i \theta}}}
$$

exists for each $\theta$. Thus, by Tauber's theorem,

$$
\sum_{n=0}^{\infty} c_{n} e^{n i \theta}
$$

converges for each $\theta$. It is easy to prove that the series is not absolutely summable (A) for $\theta=0$. 